\title{
A Modified Nelder-Mead Algorithm for Photovoltaic Parameters Identification
}

\author{
Oumaima Mesbahi *\$, Mouhaydine Tlemçani *, Fernando M. Janeiro **, Abdeloawahed Hajjaji ***, \\ Khalid Kandoussi *** \\ * ICT of Évora, University of Évora, Portugal \\ ** Instituto de Telecomunicações, Lisbon and University of Évora, Portugal \\ *** LabSIPE, University of Chouaib Doukkali, Morocco \\ (oumaimames@gmail.com, tlem@uevora.pt,fmtj@uevora.pt, hajjaji.a@ucd.ac.ma,kkandouss@gmail.com) \\ * Department of physics of Évora, University of Évora, Portugal, Tel: +351926424018, \\ oumaimames@gmail.com
}

Received: 17.12.2019 Accepted:27.01.2020

\begin{abstract}
Photovoltaic panels are prone to degradation after long outdoor exposure, which can be manifested in multiple forms. This phenomenon also causes an alteration of the panel's parameters. In this work, a heuristic search methodology based on the Nelder-Mead algorithm is applied in the estimation of photovoltaic panel parameters. Modifications that improve the performance of the method are also presented. The results of this modified Nelder-Mead algorithm are compared with the results of the classical method. A sensitivity analysis towards additive noise and ADC resolution is performed.
\end{abstract}

Keywords Photovoltaic panel, parameter estimation, Nelder-Mead algorithm, ADC, sensitivity analysis.

\section{Introduction}

The massive use of fossil fuels causes the release of huge amounts of $\mathrm{CO}_{2}$, creating an artificial and forced heating of the atmosphere. Nowadays, all the societies are engaging in a massive energy transition, focusing on adapting renewable energies [1],[2] as alternatives. This is especially true for photovoltaic (PV) energy [3], [4] that has experienced an exponential growth worldwide on recent decades.

Currently there is a lack of information on the various degradation modes [5]-[8] of photovoltaic modules in terms of frequency, speed of evolution and degree of impact on the lifetime and reliability of PV modules. In recent years, research on photovoltaic modules [2] has focused more on the the development of new technologies without having enough feedback on those that are already operational.

Although, detection methods such as infrared imaging and thermography are available, they are difficult to apply when the module is already operating.

The decrease in the produced power is the primary indicator of the degradation of the photovoltaic panel. In order to be able to detect it and its degree, the best solution is to measure its maximum power and compare it with the power value provided by the manufacturer, which only gives the information about the panel under Standard Test Conditions (STC) .

To achieve an accurate estimation of the panel parameters, researchers need to carry out experimental set-ups under well-known climatological conditions with the use of expensive instrumentation [9]. Many researchers have suggested mathematical models for parameters identification of the PV cell:

an analytical method based on the Lambert Wfunction, where the estimation of the five parameters is a multivariable and a transcendent problem, but with a limited range of calculation [10], [11].

$>$ a genetic algorithm for parameter identification has been proposed in [12], [13], however this method can be ineffective due to its binary complexity.

$>$ Particle Swarm Optimization (PSO), Artificial Bee Swarm optimization (AFS) and Shuffled Frog Leaping algorithm (SFL) methods are presented in [14]-[16], but the 
complex algorithms and long calculations lead to many uncertainties.

The aim of this work is to build a low-cost system that allows the identification of the parameters of a photovoltaic panel at any given time. This system is based on a mathematical heuristic search method known as the NelderMead algorithm. This method can be applied to any continuous function without having to evaluate its derivatives, is simple to implement, is efficient for a non-differentiable function, has an underlying geometric interpretation and ensures that the results are a decreasing series of values.

However, the classic Nelder-Mead method has some drawbacks. In addition to its dependency on the initial simplex, it applies to limitless variables and problems without constraints, stops when a local minimum is found and the search can fail by stagnation on a non-stationary point. Therefore, improvements to the Nelder-Mead method are proposed. This modified algorithm is validated by comparing its results with the existing Nelder-Mead algorithm. A sensitivity analysis towards the ADC is also presented in order to identify the optimum instrument for photovoltaic parameter identification.

\section{Photovoltaic panels}

\subsection{Degradation of photovoltaic panels}

Degradation reflects the deterioration, over time, of the characteristics of a device or a system that can reduce its capacity to operate within acceptable limits [17]. A degraded photovoltaic module should never lose its main function of generating electricity from sunshine, even if its use is no longer optimal. However, the degraded state of the photovoltaic module may be problematic when degradation exceeds a critical threshold. According to Wohlgemuth, the manufacturers consider that the PV module is degraded when its power reaches a level below $80 \%$ of its initial value [18]. In the following, different types of degradation of the most representative modules according to the literature are presented.

Photovoltaic module performance may be degraded due to several factors: temperature; humidity; irradiation; dust; and mechanical shocks [18]- [22].

Each of the different factors mentioned can induce one or more types of degradation such as discoloration; delamination; corrosion; and breaks and cracks of cells [19], [23].

Figure 1, from [24], presents the effects of the degradation, due to dust accumulation on the surface of the panel, on its I$\mathrm{V}$ characteristic.

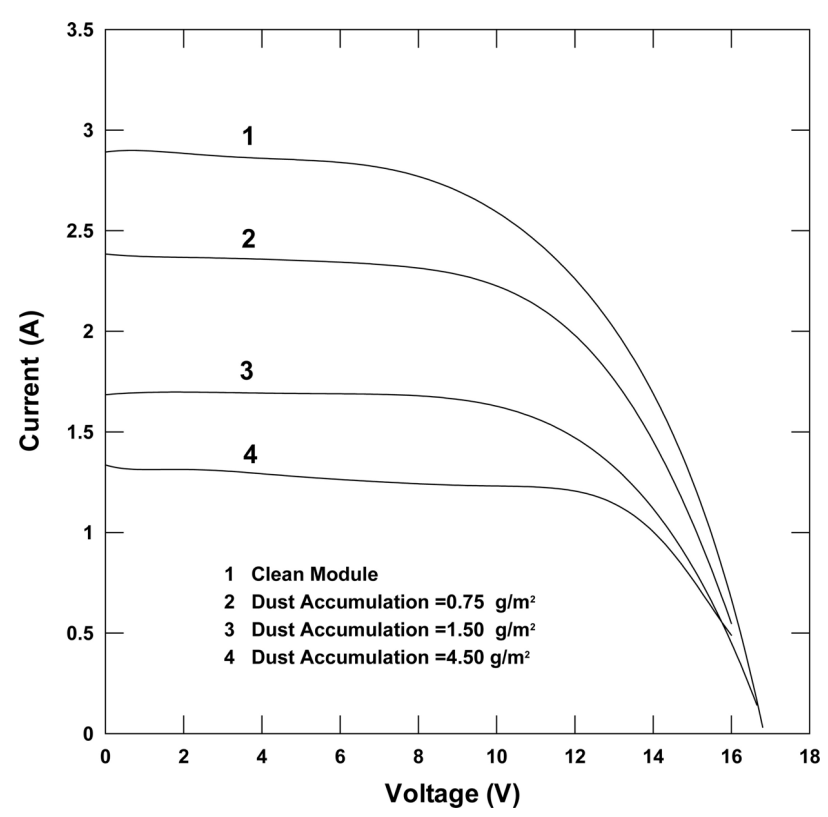

Fig. 1. The effect of accumulation dust on the I-V characteristic of a PV panel [24]

\subsection{Model of a photovoltaic cell}

In this work, the single-diode model was used for a photovoltaic cell. The single-diode model is a simple model that describes the behaviour of a solar cell. Its equivalent circuit comprises a current source of intensity proportional to the incident irradiation, in parallel with a diode and the shunt resistor $R_{S H}$ (which represents the leakage current to the ground). Internal losses due to current flow and interconnection between cells are modelled by a series resistor $R_{S}$.

The current $I$ supplied by a photovoltaic cell represented by a single diode model is

$$
I=I_{p h}-I_{S}\left[\exp \left(\frac{q\left(V+I R_{S}\right)}{n K T}\right)-1\right]-\frac{V+I R_{S}}{R_{s h}}
$$

where $V$ is the output voltage, $I_{S}$ is the saturation current, $I_{p h}$ is the photocurrent, $q$ is the electronic charge, $K$ is the Boltzmann constant, $n$ is the junction ideality factor and $T$ is the junction temperature.

The five parameters that characterize a photovoltaic panel are $I_{S}, I_{p h} I_{p h}, n, R_{S}$ and $R_{S H}$.

A photovoltaic cell has a nonlinear I-V characteristic, represented by the implicit equation (1), which includes the output current on each side of the equality, which makes it harder to solve. Therefore, the Newton-Raphson method has been used to find the roots of the (2) in order to obtain the characteristics of the PV panel. 
$f(I, V)=I-I_{p h}-I_{S}\left[\exp \left(\frac{q\left(V+I R_{s}\right)}{n K T}\right)-1\right]-\frac{V+I R_{S}}{R_{s h}}$

\section{Nelder-Mead algorithm enhancements}

\subsection{Nelder-Mead algorithm}

The Nelder-Mead algorithm is a non-linear optimization algorithm that was published first by Nelder and Mead in 1965 [25], [26]. It is a heuristic numerical method that seeks to minimize a continuous function in a multi-dimensional space. Also called downhill simplex method, the algorithm exploits the concept of simplex which is a polytope of $n+1$ vertices in a space with $n$ dimensions. The initial simplex undergoes simple transformations along the iterations: it deforms, moves and gradually shrinks until its peaks get closer to a point where the function is locally minimal.

This method uses an arrangement of $n+1$ vertices $v_{i}$ where the cost function is evaluated, where $n$ is the dimension of the search domain. A regular simplex of initial size $n+1$ is initialized in $v_{1}=x_{0}$.

Each iteration of the method starts with the vertices of a simplex and the corresponding values of the cost function. The simplex is modified through reflection, expansion, contraction, or shrinkage, and a point is accepted or rejected based on its cost function value.

A generic iteration has two possibilities: i) a new peak at least better than the worst vertex is substituted for it; otherwise, ii) a shrinking is done and a set of $n$ new vertices, plus the best of the old points, make up the simplex of the next iteration. The flowchart for Nelder-Mead's method is shown in Fig. 2. The values recommended in [25] for the reflection coefficients $(\alpha)$, contraction $(\beta)$ and expansion $(\gamma)$ are $1,1 / 2$ and 2 , respectively.

An intuitive interpretation of this algorithm is that a search direction is defined by the worst point (the one whose cost function is highest) and the centroid of all the vertices except the worst. The simplex can accelerate (expand) or decelerate (contraction) in this direction to locate an optimal region and zoom (shrink) towards the optimum point.

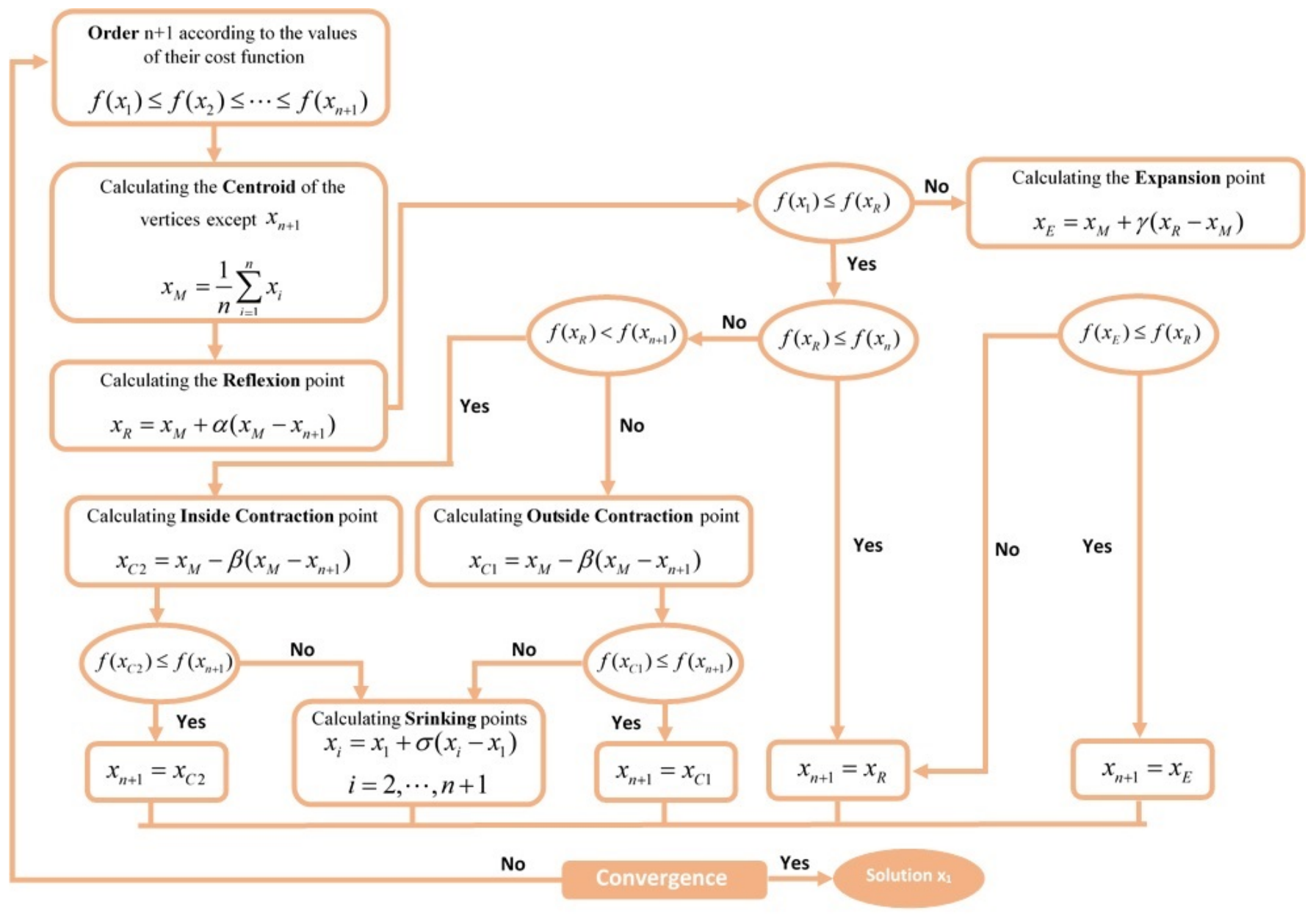

Fig. 2. Nelder-Mead algorithm flowchart 


\subsection{Convergence conditions modification}

Convergence towards a minimum point is not guaranteed by the Nelder-Mead method [27]-[29]. The evidence for convergence of one-dimensional strictly convex functions and restricted results for a set of two-dimensional functions are given in [30]. In [31] the behaviour of the method applied to a family of strictly convex and continuous two-dimensional functions up to the third derivative, where convergence (or stagnation) occurs on a non-stationary point is analysed.

In multidimensional problems, the usual convergence criteria used is the one proposed by Paul Wright in [30]. However, [30] proves the convergence of the algorithm for only one to two dimensional strictly convex functions and there are not many works concerning the convergence criteria for a multi-dimensional function. The classical convergence criteria in summarized in

$$
\left\{\begin{array}{l}
\text { NumberIteration }<\text { MaxIteration } \\
\text { NumberFunction }<\text { MaxFunction } \\
\left|v_{1}-v_{i}\right|<\text { TolX }, i=2, \cdots n+1 \\
\left|f\left(v_{1}\right)-f\left(v_{i}\right)\right|<\text { TolF }, i=2, \cdots n+1
\end{array}\right\}
$$

where MaxIteration is the maximum number of iterations and MaxFunction is the maximum number of function evaluations. The tolerances TolX and TolF bound the convergence conditions.

$>$ These conditions were tested in the estimation of the five parameters of a photovoltaic panel, under different operating conditions and it has been concluded that: the algorithm does not always converge;

$>$ the convergence condition, which is based on the difference between the worst vertex and the other ones, sometimes does not change from one iteration to the next which causes its stagnation on the remaining iterations, hence preventing the convergence of the algorithm or the convergence to an inaccurate solution.

$>$ even though this condition does not change between successive iterations, the centroid of the vertices does.

Therefore, the new convergence criterion considered taking the centroid as part of its conditions. Which will also be used to size up the shape of the simplex at each iteration. The proposed criterion is shown on the system of equations(4), which consists on: i) a maximum number of iterations; ii) a maximum number of function evaluations; iii) the maximum difference between the centroid and the vertices; and iv) the maximum difference between the cost function of the centroid and the cost function values at the vertices.

$$
\left\{\begin{array}{l}
\text { NumberIteration }<\text { MaxIteration } \\
\text { NumberFunction }<\text { MaxFunction } \\
\operatorname{Max}\left(\left|v_{M}-v_{i}\right|\right)<\text { TolX, } i=1, \cdots n+1 \\
\operatorname{Max}\left(\left|f\left(v_{M}\right)-f\left(v_{i}\right)\right|\right)<\text { TolF }, i=1, \cdots n+1
\end{array}\right\}
$$

\subsection{Initial simplex modification}

The convergence of the Nelder-Mead algorithm depends heavily on the chosen initial simplex. Choosing the first set of vertices is the initial step of the algorithm; it starts by choosing an initial approximation of the solution $x_{0}$, and then creating the rest of the vertices according to the length of the simplex. Many studies have been done regarding the generation of the simplex. Spenldey et al. presented in [32] a technique for empirical optimisation using as an initial simplex the system of equation(5). It is called the regular simplex because its edges have the same length, which means that its effectiveness will drop in the case of parameters with different orders of magnitude.

$$
v_{i}=x_{0}+p e_{i}+\sum_{\substack{k=1 \\ k \neq i}}^{n} q e_{k}, i=2, \cdots, n+1
$$

with

$$
\left\{\begin{array}{l}
p=\frac{a}{n \sqrt{2}}(\sqrt{n+1}+n-1), \\
q=\frac{a}{n \sqrt{2}}(\sqrt{n+1}-1)
\end{array}\right.
$$

To set up the initial simplex, Box presents in [33] a new randomized bounds method. This is shown in (6).

$$
v_{i}=m_{i}+r_{i}\left(M_{i}-m_{i}\right), \quad i=2, \cdots, n+1
$$

which requires the use of pseudo-random numbers and ranges for each parameter. But in this approach, the variables should be bounded, where $m_{i}$ and $M_{i}$ are respectively the minimum and the maximum bounds and $r_{i}$ is a pseudo random number chosen from the interval $[0,1]$.

Another approach, presented by Pfeffer [34], is the most widely used in the Nelder-Mead algorithm. This technique is very effective in solving the scaling problem of the variables. It starts with the initial guess for the first vertex and the rest of vertices are generated according to

$$
\left\{\begin{array}{l}
\left(v_{i}\right)_{j}=\left(x_{0}\right)_{j}+0.05 \times\left(x_{0}\right)_{j}, \quad \text { if } j=i-1 \\
\left(v_{i}\right)_{j}=\left(x_{0}\right)_{j}, \quad \text { if } j \neq i-1
\end{array}\right.
$$


with

$$
\left\{\begin{array}{l}
i=2, \cdots, n+1 \\
j=1, \cdots, n
\end{array} .\right.
$$

However, this technique of initialisation encounters some convergence problems on functions with more than two variables.

This paper presents a new approach for the initialisation of the simplex that is suited for the estimation of photovoltaic parameters which is characterised by two factors: i) multivariable function (five parameters) which makes it harder to achieve convergence; ii) the different order of magnitude of the parameters ( $I_{S}$ is of order $10^{-9}$ while $R_{S H}$ is of order $10^{2}$ ) creating a scaling problem. Taking the initial guess as first vertex, the configuration of the rest of the vertices is constructed as

$$
\left(v_{i}\right)_{j}=\left(x_{0}\right)_{j}+0.005 \times\left(x_{0}\right)_{j}
$$

with

$$
\left\{\begin{array}{l}
i=2, \cdots, n+1 \\
j=1, \cdots, n
\end{array} .\right.
$$

\section{Simulation \& Results}

\subsection{Simulation conditions}

In this work, the parameters of a photovoltaic panel are estimated using the modified Nelder-Mead algorithm. The objective of the presented simulations is to analyse the convergence of the proposed algorithm and compare the results with the ones of pre-implemented function based on the classical convergence conditions (3)and the approach suggested by Pfeffer (7)for the initial simplex. The initial guess point $x_{0}$ for the initial simplex and the bounds MaxIteration and MaxFunction are the same for both algorithms.

A sensitivity analysis of the algorithm relative to different values of additive Gaussian white noise, which is omnipresent on experimental measurements, is presented. As the measurement set-up consists on an Analog to Digital Converter (ADC), this work also presents a sensitivity analysis of the algorithm regarding the resolution of the ADC with the aim to determine the optimal number of bits for the estimation of the photovoltaic parameters.

To estimate the photovoltaic panel parameters, the Nelder-Mead algorithm is used to minimize, in a least-squares sense, the absolute difference between the ideal output current $I_{p}$ and the estimated output current $\hat{I}_{p}$ where $p \in\left\{1,2, \ldots, N_{p}\right\}$ and $N_{p}$ represents the number of current measurements

$$
S=\frac{1}{N_{p}} \sum_{p=1}^{N_{p}}\left(I_{p}-\hat{I}_{p}\right)^{2} .
$$

The ideal output current $I_{p}$ is obtained by solving (2) using the ideal parameters provided by the manufacturer in Table 1. The estimated output current is also obtained by solving (2)using the results of Nelder-Mead algorithm.

Table 1. Ideal photovoltaic parameters

\begin{tabular}{|c|c|}
\hline Parameter & Value \\
\hline$I_{p h}$ & $3.95 \mathrm{~A}$ \\
\hline$I_{S}$ & $2.16 \times 10^{-8} \mathrm{~A}$ \\
\hline$n$ & 1.2 \\
\hline$R_{S H}$ & $134.7 \Omega$ \\
\hline$R_{S}$ & $0.255 \Omega$ \\
\hline
\end{tabular}

The electrical characteristics of the photovoltaic panel used in this study are presented in Table 2.

Table 2. Electrical characteristics of the photovoltaic panel

\begin{tabular}{|c|c|c|}
\hline Parameter & Variable & Value \\
\hline Cells number & $N_{S}$ & 36 \\
\hline Maximum power & $P_{\max }$ & $60 \mathrm{~W}$ \\
\hline Voltage at $P_{\max }$ & $V_{m}$ & $17.1 \mathrm{~V}$ \\
\hline Current at $P_{\max }$ & $I_{m}$ & $3.508 \mathrm{~A}$ \\
\hline Short circuit current & $I_{s c}$ & $3.9 \mathrm{~A}$ \\
\hline Open circuit voltage & $V_{o c}$ & $21.1 \mathrm{~V}$ \\
\hline
\end{tabular}

\subsection{Noise analysis}

The first analysis concerns the estimation of five the parameters and the effect of different levels of additive white Gaussian noise. The performance of the modified NelderMead is compared with the performance of the classical algorithm, through the average and standard deviation of the estimated parameters obtained from 1000 simulations with different noise realizations. In this simulation, the standard deviation of the additive noise was varied from $2 \%$ to $20 \%$ in steps of $2 \%$. In the following results, the blue curves correspond to the proposed modified Nelder-Mead algorithm and the red curves correspond to the classical Nelder-Mead approach using (3) as convergence conditions and Pfeffer method to initialize the simplex in(7).

Figure 3 shows the mean and standard deviation values of the photocurrent $I_{p h}$ as a function of the current noise. The results show that the proposed algorithm performs better than the classical algorithm since the average value is closer to the ideal value and the standard deviation of the estimated photocurrent is also smaller indicating higher accuracy and precision. 


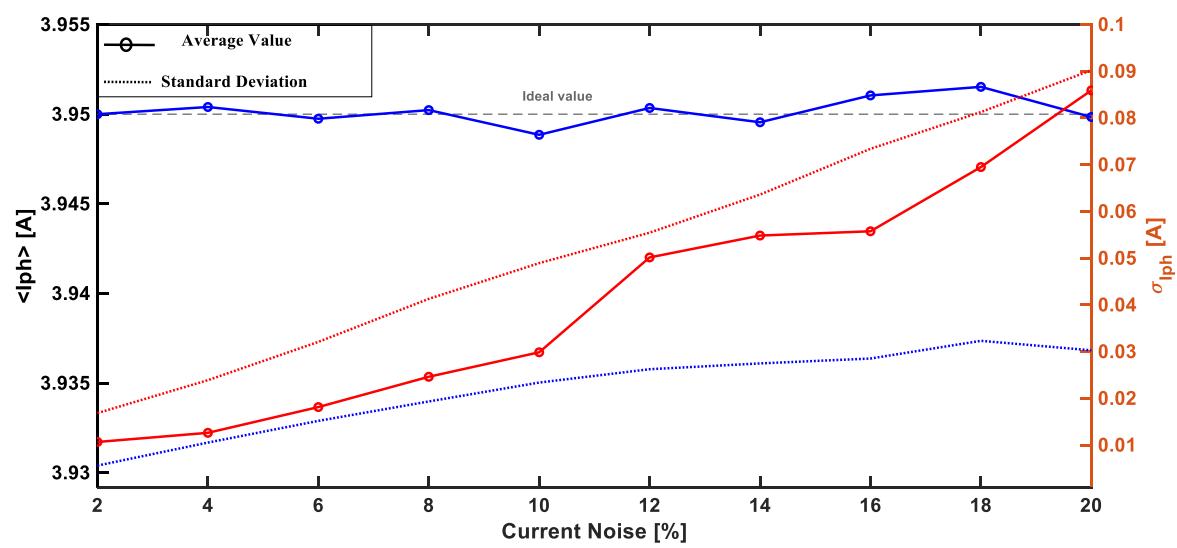

Fig. 3. Average and standard deviation of photocurrent versus current noise

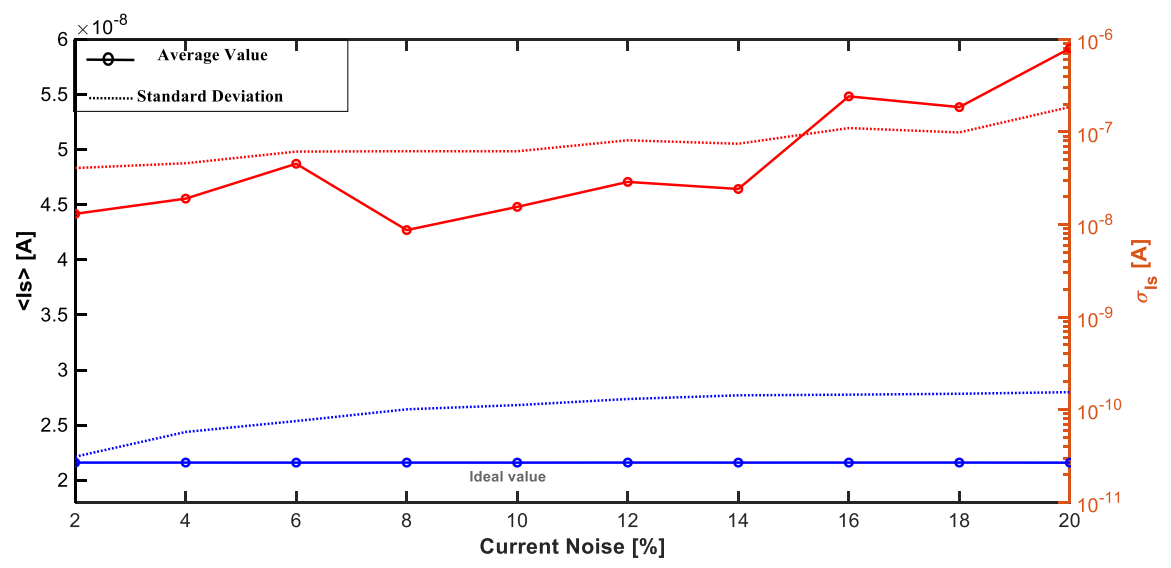

Fig. 4. Average and standard deviation of saturation current versus current noise

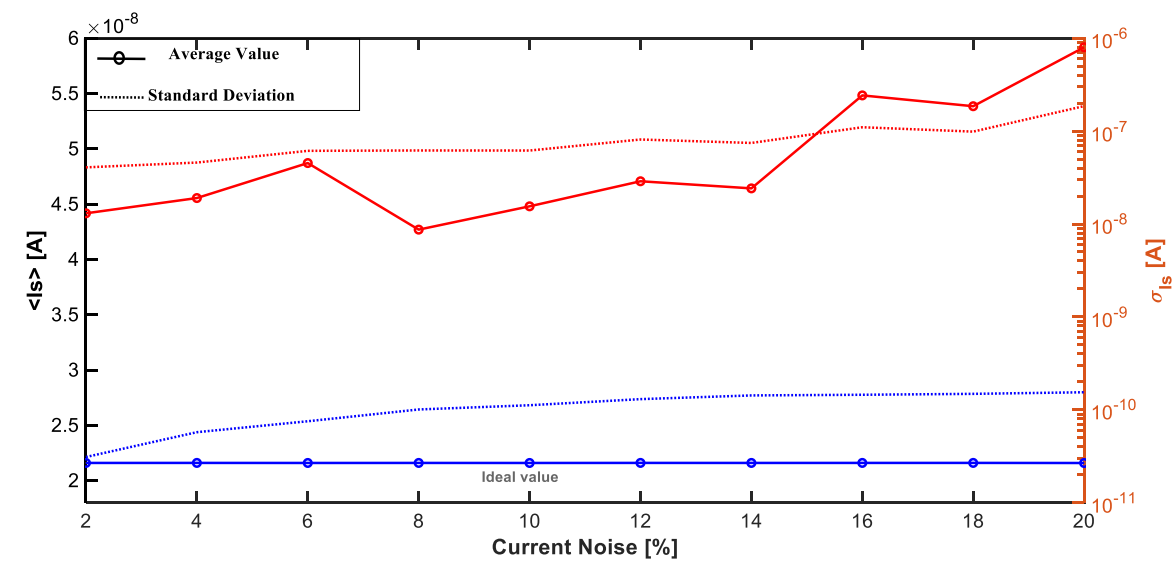

Fig. 5. Average and standard deviation of ideality factor versus current noise

Figure 4 presents the average and standard deviation of the estimated saturation current $I_{S}$. For this parameter, the classical algorithm (red curve) fails to estimate the ideal value which is around $2.16 \times 10^{-8}$ while the average results are around $4.5 \times 10^{-8}$. The modified method results (blue curve) present an average value very close to the ideal value and also a much smaller standard deviation.
Figure 5 presents the average and standard deviation of the estimated ideality factor $n$ as a function of the current noise for both algorithms. Although, for low values of noise the classical algorithm performs almost as well as the modified method on the average value, it can be seen that the standard deviation is substantially larger. 


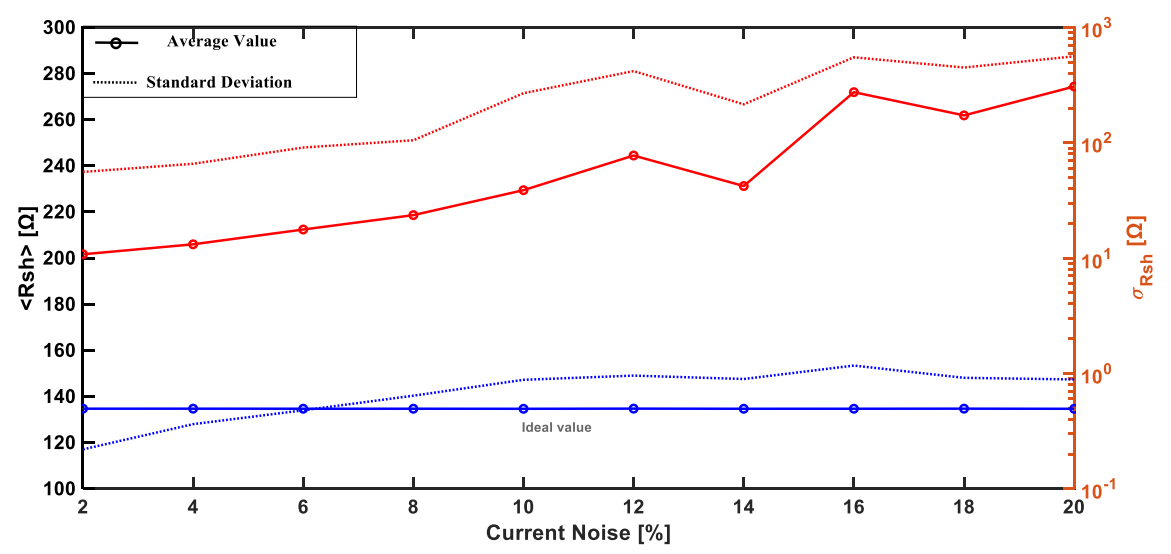

Fig. 6. Average and standard deviation of the shunt resistance versus current noise

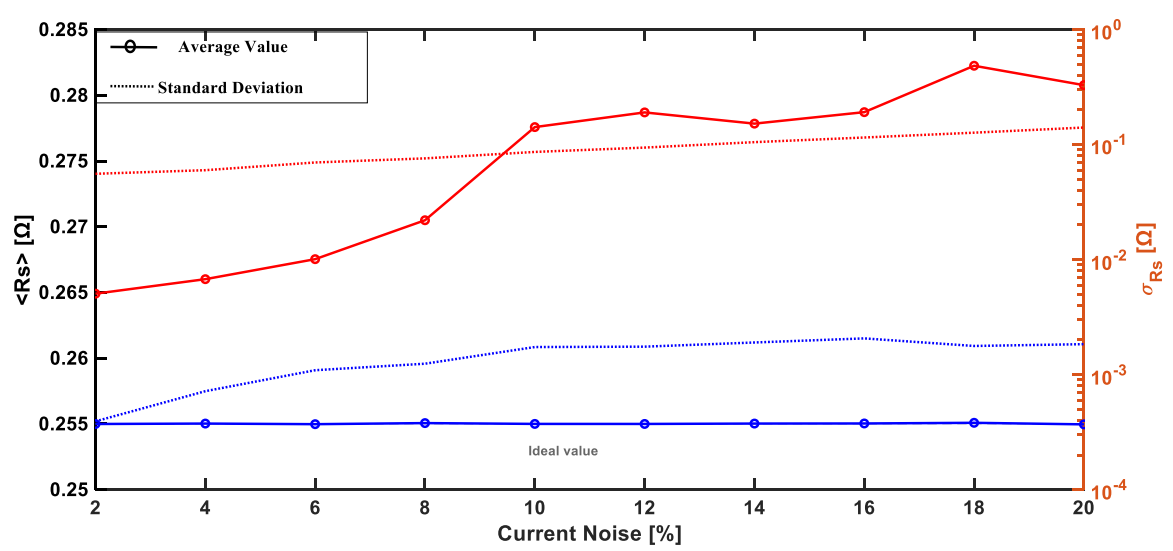

Fig. 7. Average and standard deviation of the series resistance versus current noise

Additionally, for current noise above $8 \%$ the average value of the estimated parameter starts to diverge from the ideal value.

As in the saturation current, the results obtained by the classical Nelder-Mead approach are mediocre at estimating the shunt resistance $R_{S H}$. Figure 6 shows that the red curve diverges from the ideal value even with a low percentage of noise, while the estimate made by the modified algorithm is more accurate and precise. In fact, the standard deviation of the estimated parameter by the classical method is 2 orders of magnitude higher than the standard deviation obtained with the proposed method.

Finally, Figure 7 represents the mean values and the standard deviation obtained by the two algorithms regarding the estimation of the series resistance $R_{S}$. The modified algorithm is more accurate as shown by the estimated average value and also more precise since the standard deviation of the estimated value is also much smaller than the one obtained with the classical algorithm which also fails to achieve an average value close to the ideal value.

\subsection{ADC resolution analysis}

The setup of the estimation of the photovoltaic parameters requires the use of an Analog to Digital Converter (ADC). This work models the behaviour of the ADC in order to study the effect of its resolution on the estimation of the photovoltaic parameters.

The results in Figure 8 were obtained using the modified Nelder-Mead algorithm to identify the five photovoltaic parameters from simulated voltage and current digitized by a an ideal simulated ADC. The number of bits of the ADC is in the range $[8,24]$.

Figure 8 shows the estimated parameters as a function of the number of bits of the ADC. The estimated parameters converge to the ideal values as the number of bits increase. The estimated parameters present an overall error of $0.4 \%$ for an 8 bit ADC and the results stabilize at the ideal values when the ADC has 14 or more bits. 

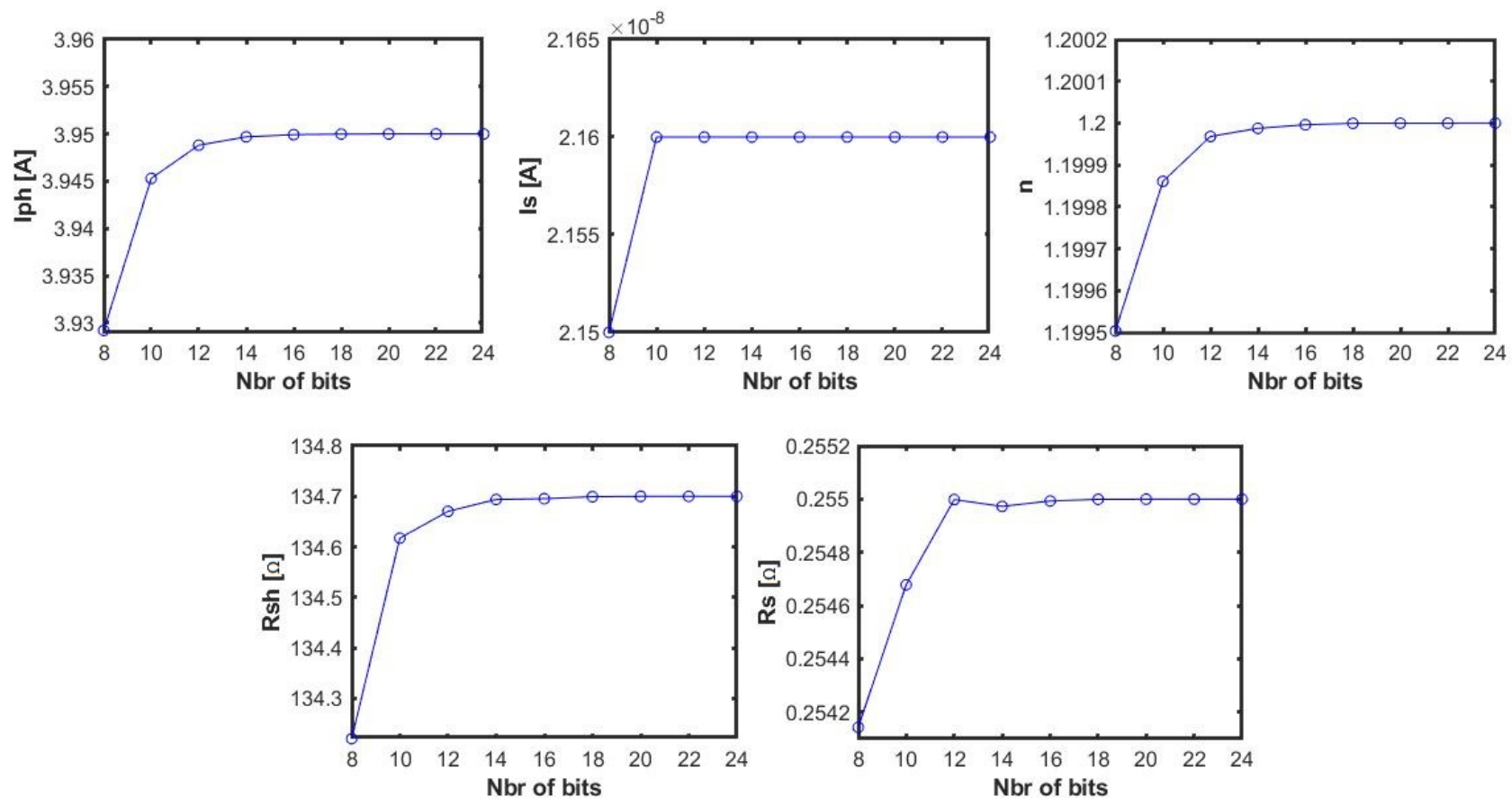

Fig. 8. Photovoltaic parameters estimation by modified Nelder-Mead algorithm versus number of bits of the ADC

\subsection{Effects of additive noise on maximum power point}

To conclude this study, the estimation of the maximum power point (MPP) has been done. Figure 9 presents the relative error of the maximum power estimated by the classical Nelder-Mead (red) and the modified Nelder-Mead (blue), under the same STD conditions $\left(1000 \mathrm{~W} / \mathrm{m}^{2}\right.$ and $\left.25^{\circ} \mathrm{C}\right)$, as a function of current noise. Figures 9 and 10 show that the error increases with noise. However, the relative error of the estimated MPP is of the order of $10^{-2} \%$ for the modified algorithm while it is between $1 \%$ and $6 \%$ when the classical method is used. The classical algorithm will provide an inaccurate estimation of the maximum power; hence it will not indicate the right degree of degradation of the photovoltaic panel. Therefore, there will be cases where it will determine that the panel is degraded but in reality the panel still has available lifetime.

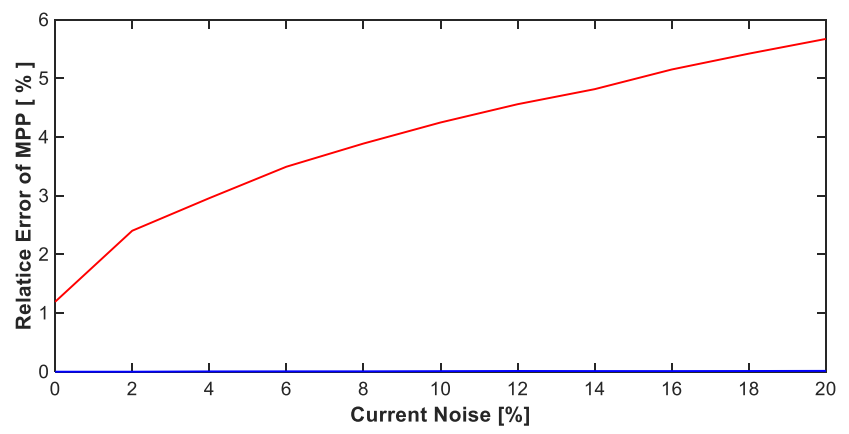

Fig. 9. Relative Error of MPP versus current noise

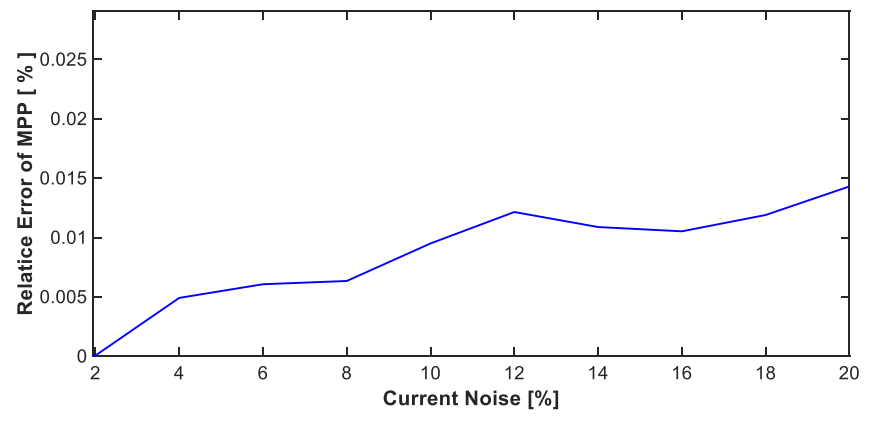

Fig. 10. Zoom of the relative error of the MPP of the modified Nelder-Mead algorithm

\section{Conclusion}

Installed photovoltaic panels sooner or later are drawn into degradation. The identification of the panel parameters gives access to its $\mathrm{I}-\mathrm{V}$ and $\mathrm{P}-\mathrm{V}$ characteristics under any given conditions. This also allows the prediction of the degree of the degradation of the panel by comparing the produced maximum power with the ideal one. This work presents new modifications to the Nelder-Mead algorithm that improve its performance regarding the estimation of the photovoltaic parameters. Comparing its results with the classical NelderMead, has shown to have more precise and accurate convergence. Even in the case of very perturbed data due to high noise, the modified algorithm has shown its ability to estimate the panel parameters with a low error. As for the $\mathrm{ADC}$, this study has concluded that a low-cost 14 bit ADC is 
suitable to address this problem. All these studies were carried out as part of the research of optimisation of the performance of the photovoltaic panels.

\section{Acknowledgements}

The authors would like to thank the ICT (Institute of Earth Sciences) of University of Évora, for the help and support to enable the work.

\section{References}

[1] Green Energy and Technology. 2012.

[2] F. Blaabjerg and D. M. Ionel, "Renewable Energy Devices and Systems-State-of-the-Art Technology, Research and Development, Challenges and Future Trends," Electr. Power Components Syst., vol. 43, no. 12 , pp. 1319-1328, 2015.

[3] U. Gangopadhyay, S. Jana, and S. Das, "State of Art of Solar Photovoltaic Technology," Conf. Pap. Energy, vol. 2013, pp. 1-9, 2013.

[4] D. Dimova-Malinovska, "The state-of-the-art and future development of the photovoltaic technologies - The route from crystalline to nanostructured and new emerging materials," J. Phys. Conf. Ser., vol. 253 , no. $1,2010$.

[5] A. Vasić, B. Loňcar, M. Vujisić, K. Stanković, and P. Osmokrović, "Aging of the photovoltaic solar cells," 2010 27th Int. Conf. Microelectron. MIEL 2010 - Proc., no. Miel, pp. 487-490, 2010.

[6] A. K. Tripathi, M. Aruna, and C. S. N. Murthy, "Output Power Loss of Photovoltaic Panel Due to Dust and Temperature," Int. J. Renew. ENERGY Res., vol. 7, no. 1, pp. 1-4, 2017.

[7] R. C. Moura, M. M. F. Gemignani, C. F. M. Almeida, and N. Kagan, "Analysis of losses by soiling and degradation: Methodological proposal for applications in situ," in 2017 IEEE 6th International Conference on Renewable Energy Research and Applications (ICRERA), 2017, pp. 484-490.

[8] N. M. Kumar, N. Prabaharan, A. R. A. Jerin, and A. Jayakumar, "Impact of Performance Degradation and Capital Subsidy on the Revenue of Rooftop PV System," Int. J. Renew. ENERGY Res., vol. 9, no. 1, 2019.

[9] M. Hassan Ali, A. Rabhi, S. Haddad, and A. El Hajjaji, "Real-Time Determination of Solar Cell Parameters," J. Electron. Mater., vol. 46, no. 11, pp. 6535-6543, 2017.

[10] M. Taciuc and A. Crăciunescu, "Application of the Lambert W-function for a PV module parameters' estimation," AIP Conf. Proc., vol. 1863, 2017.

[11] A. Jain and A. Kapoor, "Exact analytical solutions of the parameters of real solar cells using Lambert Wfunction," Sol. Energy Mater. Sol. Cells, vol. 81, no.
2, pp. 269-277, 2004.

[12] A. M. Dizqah, A. Maheri, and K. Busawon, "An accurate method for the PV model identification based on a genetic algorithm and the interior-point method," Renew. Energy, vol. 72, pp. 212-222, 2014.

[13] M. Zagrouba, A. Sellami, M. Bouaïcha, and M. Ksouri, "Identification of PV solar cells and modules parameters using the genetic algorithms: Application to maximum power extraction," Sol. Energy, vol. 84, no. 5, pp. 860-866, 2010.

[14] A. Askarzadeh and A. Rezazadeh, "Artificial bee swarm optimization algorithm for parameters identification of solar cell models," Appl. Energy, vol. 102, pp. 943-949, 2013.

[15] J. J. Soon and K. S. Low, "Photovoltaic model identification using particle swarm optimization with inverse barrier constraint," IEEE Trans. Power Electron., vol. 27, no. 9, pp. 3975-3983, 2012.

[16] N. Tutkun, E. Elibol, and M. Aktaş, "Parameter extraction from a typical PV module using a metaheuristic technique," in 2015 International Conference on Renewable Energy Research and Applications (ICRERA), 2015, pp. 755-759.

[17] A. Lannoy and H. Procaccia, Evaluation et maîtrise $d u$ vieillissement industriel. 2005.

[18] J. H. Wohlgemuth, D. W. Cunningham, P. Monus, J. Miller, and A. Nguyen, "Long term reliability of photovoltaic modules," in 2006 IEEE 4th World Conference on Photovoltaic Energy Conference, 2006, vol. 2, pp. 2050-2053.

[19] M. A. Munoz, M. C. Alonso-García, N. Vela, and F. Chenlo, "Early degradation of silicon PV modules and guaranty conditions," Sol. energy, vol. 85, no. 9, pp. 2264-2274, 2011.

[20] A. Ndiaye, A. Charki, A. Kobi, C. M. F. Kébé, P. A. Ndiaye, and V. Sambou, "Degradations of silicon photovoltaic modules: A literature review," Sol. Energy, vol. 96, pp. 140-151, 2013.

[21] C. R. Osterwald and T. J. McMahon, "History of accelerated and qualification testing of terrestrial photovoltaic modules: A literature review," Prog. Photovoltaics Res. Appl., vol. 17, no. 1, pp. 11-33, 2009.

[22] M. A. Quintana, D. L. King, T. J. McMahon, and C. R. Osterwald, "Commonly observed degradation in field-aged photovoltaic modules," in Conference Record of the Twenty-Ninth IEEE Photovoltaic Specialists Conference, 2002., 2002, pp. 1436-1439.

[23] M. Vázquez and I. Rey-Stolle, "Photovoltaic module reliability model based on field degradation studies," Prog. photovoltaics Res. Appl., vol. 16, no. 5, pp. 419-433, 2008.

[24] A. Y. Al-hasan and A. A. Ghoneim, “A new 
correlation between photovoltaic panel's efficiency and amount of sand dust accumulated on their surface," Int. J. Sustain. Energy, vol. 24, no. 4, pp. 187-197, Dec. 2005.

[25] J. A. Nelder and R. Mead, "A simplex method for function minimization," Comput. J., vol. 7, no. 4, pp. 308-313, 1965.

[26] J. C. Lagarias, J. C. Lagarias, J. A. Reeds, M. H. Wright, and P. E. Wright, "Convergence Properties of the Nelder-Mead Simplex Method in Low Dimensions," Siam J. Optim., vol. 9, no. 1, pp. 112 147, 1998.

[27] T. G. Kolda, R. M. Lewis, and V. Torczon, "Optimization by direct search: New perspectives on some classical and modern methods," SIAM Rev., vol. 45 , no. 3 , pp. 385-482, 2003.

[28] R. M. Lewis, V. Torczon, and M. W. Trosset, "Direct search methods: then and now," J. Comput. Appl. Math., vol. 124, no. 1, pp. 191-207, 2000.

[29] C. T. Kelley, "Detection and Remediation of
Stagnation in the Nelder--Mead Algorithm Using a Sufficient Decrease Condition," SIAM J. Optim., vol. 10, no. 1, pp. 43-55, 1999.

[30] J. C. Lagarias, J. A. Reeds, M. H. Wright, and P. E. Wright, "Convergence properties of the Nelder-Mead simplex method in low dimensions," SIAM J. Optim., vol. 9, no. 1, pp. 112-147, 1998.

[31] K. I. M. McKinnon, "Convergence of the Nelder-Mead Simplex Method to a Nonstationary Point," SIAM J. Optim., vol. 9, no. 1, pp. 148-158, 1998.

[32] W. Spendley, G. R. Hext, and F. R. Himsworth, "Sequential application of simplex designs in optimisation and evolutionary operation," Technometrics, vol. 4, no. 4, pp. 441-461, 1962.

[33] M. J. Box, "A new method of constrained optimization and a comparison with other methods," Comput. J., vol. 8, no. 1, pp. 42-52, 1965.

[34] "Global optimization of lennard-jones atomic clusters." 